\title{
Patterns of Potential Magnetic Field Merging Sites on the Dayside Magnetopause
}

\author{
J. G. Luhmann, ${ }^{1}$ R. J. Walker, ${ }^{1}$ C. T. Russell, ${ }^{1}$ N. U. Crooker, ${ }^{2}$ \\ J. R. Spreiter, ${ }^{3}$ and S. S. Stahara ${ }^{4}$
}

\begin{abstract}
Models of the magnetospheric and magnetosheath magnetic fields are used to determine the relative orientations of these fields at the dayside magnetopause in order to locate potential merging sites. Areas on the magnetopause with different fractional antiparallel components are displayed by contour diagrams for a variety of interplanetary field orientations. For interplanetary fields oriented perpendicular to the solar wind velocity the areas of nearly antiparallel field agree with those obtained by Crooker using simplified representations for the magnetic field geometry. Here, the application of more realistic models gives the locations of areas where any antiparallel component occurs. Potential merging sites for interplanetary fields with radial components are also illustrated. The results suggest that the topology of the magnetosheath and magnetospheric fields provides antiparallel components over a substantial fraction of the magnetopause for most interplanetary field orientations.
\end{abstract}

\section{INTRODUCTION}

Dungey [1961] was the first to propose that the merging or reconnection of the interplanetary and terrestrial magnetic fields where they are antiparallel was responsible for coupling solar wind energy into the magnetosphere. The reconnection process could transfer magnetic flux from the dayside magnetosphere to the magnetotail when the interplanetary field was directed southward and from the tail to the dayside magnetosphere when it was northward [Dungey, 1963]. The evidence that this mechanism is important comes from the responses of the magnetopause and magnetotail to the southward interplanetary field [cf. Russell and McPherron, 1973] and the dependence of geomagnetic activity on the interplanetary field orientation [cf. Burton et al., 1975].

Until recently it has been difficult to examine the merging process at the magnetopause because of both a lack of adequate instrumentation and a lack of understanding of the signatures of reconnection in the data. However, the ISEE 1 and 2 spacecraft have now returned measurements that have been interpreted as observations of steady reconnection [Paschmann et al., 1979; Sonnerup et al., 1981] and patchy reconnection [Russell and Elphic, 1979]. The response of these particle and field signatures to the interplanetary field orientation can provide tests of various hypotheses concerning the locations of the reconnecting regions. A purely southward interplanetary field produces a line of perfectly antiparallel magnetospheric and magnetosheath fields near the geomagnetic equator, but sites of antiparallel field for other interplanetary field orientations are less easily visualized. Moreover, it has been pointed out [cf. Cowley, 1976] that merging can occur where the two fields are not exactly antiparallel.

\footnotetext{
'Institute of Geophysics and Planetary Physics, University of California, Los Angeles, CA 90024.

2 Department of Atmospheric Sciences, University of California, Los Angeles, CA 90024.

${ }^{3}$ Department of Applied Mechanics, Stanford University, Stanford, CA 94305.

${ }^{4}$ Neilsen Engineering and Research, Inc., Mountain View, CA 94043.
}

Copyright 1984 by the American Geophysical Union.

Paper number 3A1904.

0148-0227/84/003A-1904\$02.00
Several years ago, Crooker [1979] presented a qualitative model of the sites of magnetic field merging on the magnetopause. This analysis was based on a conceptual picture of the magnetopause and a superposed overlying uniform field, perpendicular to the earth-sun line, that was taken to represent the magnetosheath field at its inner boundary. The latter was rotated to mimic the effect of different interplanetary field directions. Reconnection or merging lines were defined as the locus of points for which these approximate magnetospheric and magnetosheath fields were antiparallel when projected onto a plane (the GSE $Y Z$ plane). The major point arising from this analysis was the role of the cusp in locating potential merging sites. The present study extends the ideas put forth in this earlier effort by employing more realistic models of the magnetospheric and magnetosheath fields to locate antiparallel fields at their magnetopause interface. A gasdynamic magnetosheath model with frozen field is used to describe the draping of the interplanetary field over the magnetospheric obstacle. The new aspects of this analysis include the location of fields that are not exactly antiparallel and the treatment of interplanetary fields with radial (GSE $X$ ) components that were not considered previously.

\section{DESCRIPTION OF THE MODEL}

The Hedgecock and Thomas model [Walker and Southwood, 1982, and references therein] was selected to represent the magnetospheric field at the dayside magnetosheath. This empirical model, which is similar to the earlier model of Mead and Fairfield [1975], was constructed from a large amount of spacecraft magnetometer data that were sorted according to magnetospheric activity and season. The model has a fairly accurate cusp geometry in the northern hemisphere, where most of the data were obtained. Because of this bias a mirror image of the model north of the equator was used to represent the southern magnetosphere. Field lines in the noon-midnight plane are shown on the right side of Figure 1. The dipole axis of the model was made to coincide with the GSE $Z$ axis for this study. Although the seasonal variation of the dipole tilt with respect to this axis will modify the magnetospheric field at the magnetopause, the complication introduced by the use of tilted models with asymmetrical magnetopause shapes precluded the study of this effect. (The magnetosheath model assumes an axially symmetric obstacle.)

The magnetosheath field was modeled by using a gasdyna- 


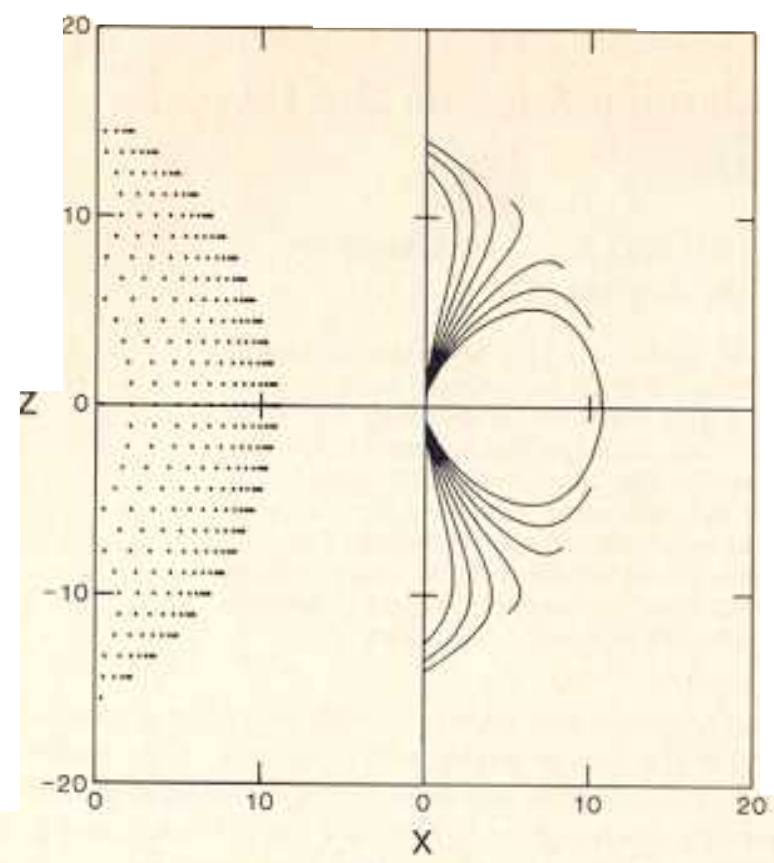

Fig. 1. A view in the GSE $X Z$ plane of the grid points on the Hedgecock-Thomas model magnetopause at which the dot product of the magnetospheric field model and the gasdynamic magnetosheath field model was computed. Only points sunward of the terminator plane were considered in this study. Also shown on the ight are the magnetospheric model field lines in the noon meridian.

mic treatment that assumes the interplanetary field is frozen into the supersonic solar wind flowing around an axisymmetric obstacle with monotonically increasing radial dimension [cf. Spreiter and Stahara, 1980]. For the present analysis the shape of the obstacle was derived from the shape of the Hedgecock and Thomas model magnetopause in the noonmidnight meridian. In the calculation of the gasdynamic flow field the aberration of the solar wind flow was neglected, and a free-stream sonic Mach number of 6.0 was used. Because the field determined with the gasdynamic code becomes inaccurate near the stagnation streamline that bathes the obstacle surface [cf. Alksne and Webster, 1970], the model field component tangential to the obstacle surface at a distance of 0.5 earth radii from the obstacle was taken to represent the magnetosheath field at the magnetopause. This approximation, which presumes that the direction of the magnetosheath field does not change very much within $\sim 0.5$ earth radii of the magnetopause, was validated by testing for changes in the tangential field direction at several other distances.

The angle between the two modeled fields was found over the surface of the dayside magnetopause at the grid points shown in the left panel of Figure 1. Contour diagrams of the cosine of this angle were then constructed to display the regions of varying antiparallel orientations as viewed from the sun.

\section{RESULTS}

Figure 2 contains the contour diagrams described above for various interplanetary magnetic field orientations (i.e., magnetosheath models). The shaded areas, representing fields that are within $10^{\circ}$ of being antiparallel, are roughly consistent with Crooker's [1979] merging lines for interplan- etary field orientations perpendicular to the solar wind velocity. New sites arise when there is a substantial GSE $X$ or radial component of the interplanetary field. As expected, the southward directed interplanetary field produces the largest area of nearly antiparallel fields on the magnetopause, while the northward interplanetary field produces the smallest area. However, the regions for which there is some antiparallel component of the magnetosphere and magnetosheath fields cover a large region of the magnetopause for most interplanetary field orientations.

\section{Discussion AND ConClusions}

For several reasons the patterns shown in Figure 2 must be considered with some measure of caution when comparing with the locations of observational evidence of merging at the magnetopause. First, because the interplanetary field is quite variable, the field measured by a spacecraft in the solar wind must be propagated to the magnetopause with the proper time lag. Except under circumstances of exceptionally steady interplanetary field orientation, the actual pattern of antiparallel fields projected on the magnetopause will not usually reflect the field observed by the same spacecraft in the solar wind at an earlier time. Second, currents in the boundary layer and MHD effects in the magnetosheath, such as those described by $Z$ wan and Wolf [1976], will affect the field geometry at the magnetopause. Third, the possible dependence of the merging rate on the local plasma velocity [Mitchell and Kan, 1978] has not been folded into these patterns. If slow flow is necessary for merging, regions of antiparallel field near the subsolar stagnation point will be favored. Similarly, the possible dependence of the merging rate on the field magnitudes has not been considered here. Finally, reconnection itself may produce a field geometry where the reconnected field lines appear to be pulled over the magnetopause [cf. Crooker, 1979], disrupting any pattern imposed purely by the magnetospheric and magnetosheath fields. (The boundary layer may be a manifestation of the latter process.) Yet the patterns shown in Figure 2 may provide a picture of the initial conditions set up by a sudden change in interplanetary field orientation.

Russell and Elphic [1979] introduced the term "flux transfer events" to describe their observations of distinctive features in the magnetic field at the magnetopause during periods of southward interplanetary field. According to these authors, a flux transfer event is produced when a localized merging of magnetosheath and magnetospheric fields takes place. The reconnected field lines twist into a ropelike structure. The magnetosheath and magnetospheric fields bulge outward from the magnetopause as the flux rope is pulled through under the combined forces of magnetic stresses and antisunward magnetosheath convection. Recent statistical studies of the spatial distribution of flux transfer events near the magnetopause (J. Berchem and C. T. Russell, unpublished manuscript, 1983) show a double-banded latitudinal distribution that flanks the equator. These authors have argued that their results are consistent with the preferred occurrence of reconnection at the equator where quasi-steady merging has been predicted for southward interplanetary field [Dungey, 1961; Sonnerup, 1976]. However, their distributions are also consistent with a superposition of the patterns for southward fields and for GSE $Y$ fields as shown in Figure 2. In fact, in their study, some events occurred in association with practically GSE $Y$ directed 


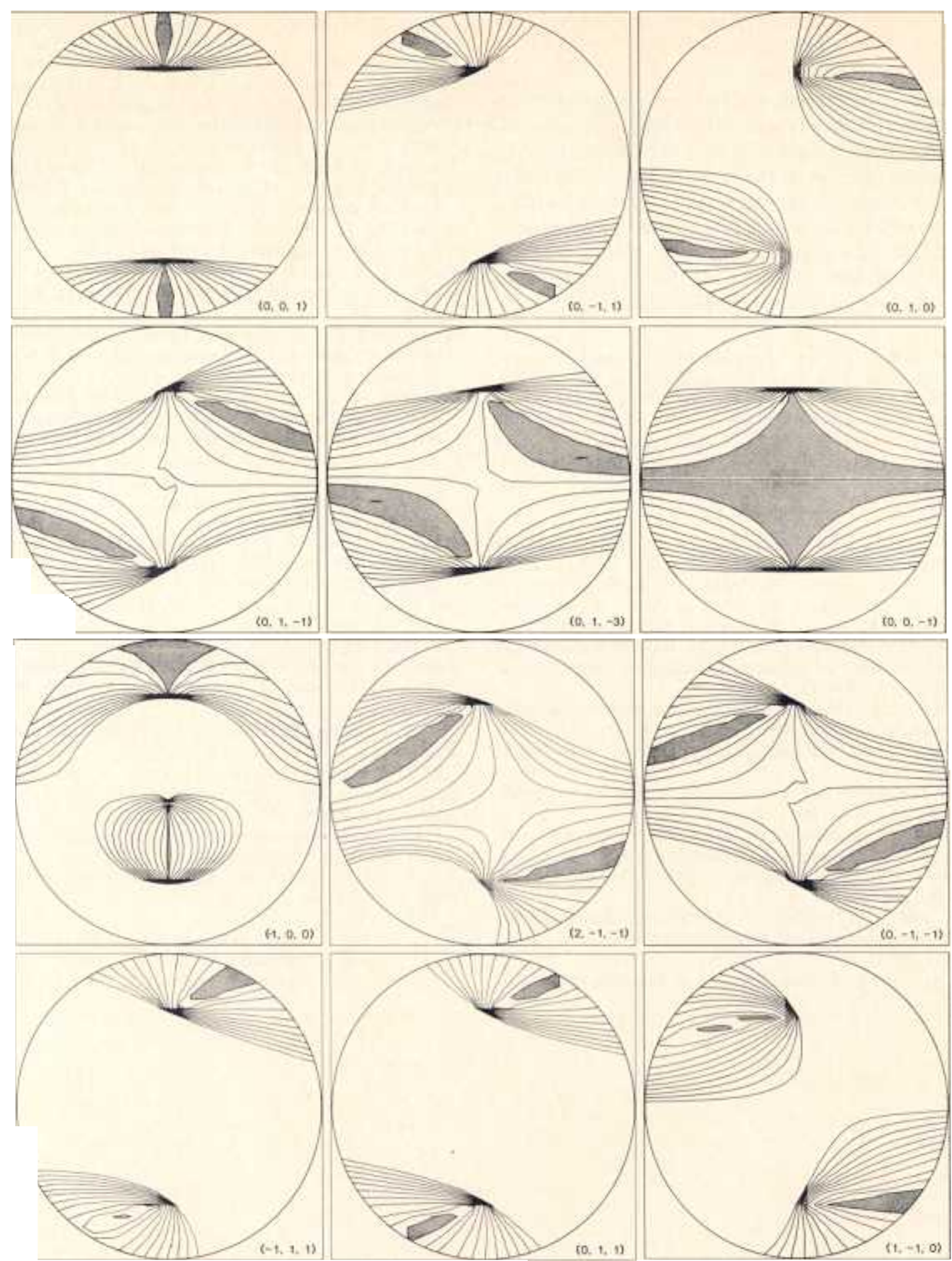

Fig. 2. Contours on the dayside magnetopause (viewed from the sun, i.e., the GSE $Y Z$ plane projection) of equal value of the cosine of the angle between the magnetospheric and magnetosheath model fields. Only contours with negative values, implying some antiparallel component, are shown. Values at the contours, starting with the contour filled with shading, are $-0.98,-0.95,-0.9,-0.8,-0.7,-0.6,-0.5,-0.4,-0.3,-0.2,-0.1,0$. The interplanetary field orientations are indicated in vector notation $\left(B_{x}, B_{y}, B_{z}\right)$ in the lower right corners. In particular, the patterns for northward and southward fields occupy the first position in the left column and the second position in the right column, respectively. The pattern for a $45^{\circ}$ cone angle is shown in the third position of the middle column.

fields. Also, because the highest geomagnetic latitudes were not covered in their sample, the poleward merging of the cusps predicted for northward directed fields (see Figure 2) could not have been observed. Still, separation of their data into events associated with steady, primarily southward, interplantary fields and GSE $Y$ directed fields may produce the distinctive patterns shown in Figure 2 for these two cases. Provided that the flux transfer events are observed near the location where they are produced by merging, one would expect the GSE $Y$ fields to give events in opposing corners along a diagonal through the subsolar point, while southward fields would give a broad latitudinal band, includ- 
ing the equator. A second study of flux transfer events by Rijnbeek et al. [1983] finds occurrences at the equator if the selection criterion is relaxed.

In conclusion, the present analysis represents the extent to which one can practically go in using steady magnetic field models to understand the global picture of reconnection sites on the magnetopause, given the present state of computing resources and theory. Time-dependent, three-dimensional MHD models of the solar wind interaction with the magnetosphere, such as that developed by $W u$ et al. [1981] and Brecht et al. [1982] will, of course, be the ultimate theoretical tool for the investigation of merging sites.

Acknowledgments. The authors would like to thank J. Berchem for useful discussions. This work was supported by National Science Foundation grant ATM-82-10691. One of the authors (JRS) acknowledges support by NAS grant NAGW 278, another (SSS) acknowledges grant NASW 3791 . The Editor thanks N. Sckopke and another referee for their assistance in evaluating this paper.

\section{REFERENCES}

Alksne, A. Y., and D. L. Webster, Magnetic and electric fields in the magnetosheath, Planet. Space Sci., 18, 1203, 1970.

Brecht, S. H., J. G. Lyon, J. A. Fedder, and K. Hain, A timedependent three-dimensional simulation of the earth's magnetosphere: Reconnection events, J. Geophys. Res., 87, 6098, 1982.

Burton, R. K., R. L. McPherron, and C. T. Russell, An empirical relationship between interplanetary conditions and Dst, J. Geaphys. Res., 80, 4204, 1975.

Cowley, S. W. H., Comments on the merging of nonantiparallel magnetic fields, J. Geophys. Res., 81, 3455, 1976.

Crooker, N. U., Dayside merging and cusp geometry, J. Geophys. Res., 84, 951, 1979.

Dungey, J. W., Interplanetary magnetic field and the auroral zone, Phys. Rev. Lett., 6, 47, 1961.

Dungey, J. W., The structure of the exosphere, or adventures in velocity space, in Geophysics of the Earth's Environment, edited by C. DeWitt, J. Hieblot, A. Lebeau, pp. 505-550, Gordon and Breach, N.Y., 1963.

Mead, G. D., and D. H. Fairfield, A quantitative magnetospheric model derived from spacecraft magnetometer data, J. Geophys. Res., 80, 523, 1975.

Mitchell, H. G., and J. R. Kan, Merging of magnetic fields with field-aligned plasma flow components, J. Plasma Phys., 20, 31, 1978.

Paschmann, G., B. U. O. Sonnerup, I. Papamastorakis, N. Sckopke, G. Haerendel, S. J. Bame, J. B. Asbridge, J. T. Gosling, C. T. Russell, and R. C. Elphic, Plasma acceleration at the earth's magnetopause: Evidence for reconnection, Nature, 282, 243, 1979.

Rijnbeek, R. P., S. W. H. Cowley, D. J. Southwood, and C. T. Russell, A survey of dayside flux transfer events observed by ISEE 1/2 magnetometers, J. Geophys. Res., in press, 1983.

Russell, C. T., and R. L. McPherron, The magnetotail and substorms, Space Sci. Rev., 15, 205, 1973.

Russell, C. T., and R. C. Elphic, ISEE observation of flux transfer events at the dayside magnetopause, Geophys. Res. Lett., 6, 33, 1979.

Sonnerup, B. Ü. O., Magnetopause and boundary layer, in Physics of Solar Planetary Environments, edited by D. J. Williams, AGU, Washington, D. C., 1976.

Sonnerup, B. Ü. O., G. Paschmann, I. Papamastorakis, N. Sckopke, G. Haerendel, S. J. Bame, J. R. Asbridge, J. T. Gosling, and $\mathrm{C}$. T. Russell, Evidence for magnetic field reconnection at the earth's magnetopausê, J. Geophys. Res., 86, 10049, 1981.

Spreiter, J. R., and S. S. Stahara, A new predictive model for determining solar wind-terrestrial planet interactions, $J$. Geophys. Res., 85, 6769, 1980.

Walker, R. J., and D. S. Southwood, Momentum balance and flux conservation in model magnetospheric magnetic fields, $J$. Geophys. Res., 87, 7460, 1982.

Wu, C. C., R. J. Walker, and J. M. Dawson, A three-dimensional MHD model of the earth's magnetosphere, Geophys. Res. Lett., $8,525,1981$.

Zwan, B. J., and R. A. Wolf, Depletion of solar wind plasma near a planetary boundary, J. Geophys. Res., 81, 1636, 1976.

N. U. Crooker, Department of Atmospheric Sciences, University of California, Los Angeles, CA 90024.

J. G. Luhmann, R. J. Walker, and C. T. Russell, Institute of Geophysics and Planetary Physics, University of California, Los Angeles, CA 90024.

J. R. Spreiter, Department of Applied Mechanics, Stanford University, Stanford, CA 94305.

S. S. Stahara, Neilsen Engineering and Research, Inc., Mountain View, CA 94043.

(Received August 17, 1983; revised November 11, 1983; accepted November 30, 1983. 